\title{
Thermal Design of Large Storage Vessels for "Liquid Hydrogen and Helium ${ }^{1}$
}

\begin{abstract}
Russell B. Scott
Principles of insulating vessels for the storage and transportation of liqueried gases are discussed. The use of shields cooled by the vapor leaving the liquid being stored is analyzed, and formulas, graphs, and tables are presented for the convenience of the designer of large vessels for storing and transporting liquid hydrogen and liquid helium. An analysis is given of the use of escaping vapor to intercept heat entering by conduction through solid supporting members. The evidence presented indicates that substantial improvements are possible in the design of such vessels.
\end{abstract}

\section{Introduction}

The current expansion in large-scale production of liquid hydrogen and helium has increased the importance of efficient storage and transport vessels for these expensive liquids. Published information on the design of low-loss containers includes descriptions $[1,2,3,4,5,6]^{2}$ of small laboratory vessels designed for storage of liquid helium. These, of course, are suitable also for storing liquid hydrogen. Some large, rugged, transportable liquid-hydrogen vessels have been constructed but have not yet been described in the open literature. It is the purpose of this paper to discuss some of the general principles relating to the thermal design of low-loss vessels and to present analyses of methods that can be used to improve the performance of an insulated vessel by utilizing the refrigerative effect of the escaping vapor. The material presented will relate specifically to the design of large vessels for liquid hydrogen and helium, although the principles used can be employed in the design of vessels for other liquefied gases.

The invention of the vacuum-insulated vessel for liquefied gases by James Dewar in 1892 was a breakthrough in the field of thermal insulation that has not yet been matched by further developments. All of the advances since Dewar's time have been improvements on Dewar's original concept, usually means of reducing radiant-heat transfer by attaining surfaces of higher reflectivity or by interposing shields that reflect or intercept the radiant energy. The Dewar vessel is illustrated in figure 1. At its best, the vacuum is so good that heat transfer by residual gas is almost negligible. Accordingly, the designer is mainly concerned with reducing the heat transferred by mechanical supports or conveyed across the insulating vacuum by radiation. The heat transferred by the supports is a problem involving the mechanical strength required for a specific application and has no general solution. If there is no restriction on the dimensions of the vessel, the heat conducted through the supports can usually be made quite small simply by using supports of low thermal conductivity and making their lengths great enough.

1 This work was supported by the U.S. Atomic Energy Commission.

2 Figures in brackets indicate the literature references at the end of this paper.
Even in restricted space the clever designer can usually find a way to provide a long thermal path through the solid supports. On the other hand, the heat transferred by radiation is little affected by the thickness of the insulating space. However, this latter mode of heat transfer can be reduced by interposing thermal shields between the warm and cold surfaces of the vessel. The shield is an opaque, highly reflecting surface, geometrically similar to the surface of the liquid container and suspended with the minimum of thermal contact approximately midway between the inner and outer walls of the vacuum space. If the shield has the same reflectivity as that of the boundary walls and contains no openings or irregularities, the radiant-heat transfer will be re-

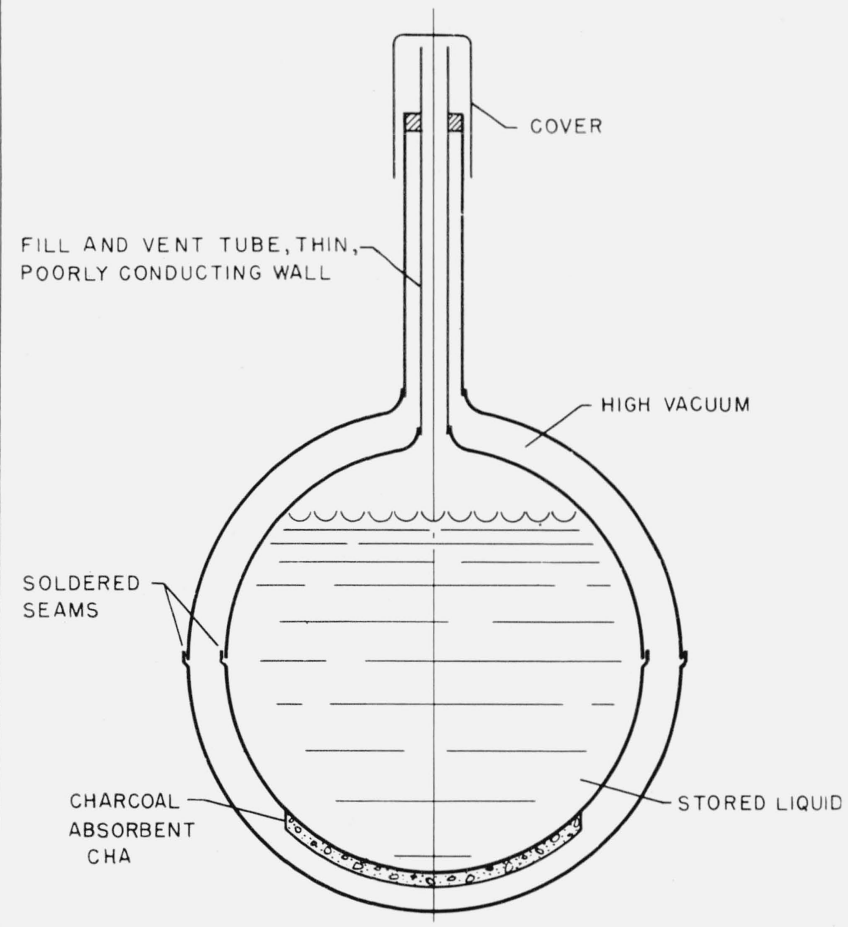

Figure 1. Metal Dewar vessel.

Commercial vessels of this type usually are mounted in an outer protective shell of sheet steel. 
duced by about one-half. Additional shields will further reduce the heat transferred by radiation. However, the design of mechanical supports, which will hold the shields in place without providing objectionable heat leaks, becomes a formidable task as the number of shields is increased. Also, openings and irregularities that are needed to accommodate the piping and supports for the inner container greatly reduce the effectiveness of this type of multiple shielding.

\section{Evacuated-Powder Insulation}

Fortunately, a method has been discovered that does not complicate the mechanical design and yet provides a barrier to thermal radiation comparable with multiple shields. This method consists of filling the insulating vacuum space with a fine light powder, such as perlite, sil ca-aerogel, carbon black, or diatomaceous earth. If the thickness of the insulating space is sufficient, filling with powder will greatly reduce the radiant-heat transfer. Moreover, the vacuum requirement is quite modest; the minimum heat transfer is very closely approached when the interstitial gas pressure is reduced to $10 \mu$ of mercury in an insulating powder (e.g., perlite or silica-aerogel) with one boundary at room temperature, $300^{\circ} \mathrm{K}$, and the othar at the temperature of liquid nitrogen, $77^{\circ} \mathrm{K}$. A layer of evacuated powder several centimeters thick will effectively stop radiation and yet not provide an objectionable amount of thermal conduction through the touching particles of powder. When the space between the walls must be small, of the order of $1 \mathrm{~cm}$, the thermal conduction through the powder is objectionable, so in this case the most effective insulation is a high vacuum bounded by highly reflecting walls. Because the principal function of the powder is to stop radiation, it is useful only when radiation constitutes an important heat leak. If an insulating vacuum is bounded by highly reflecting surfaces, such as clean annealed copper, silver, or aluminum, and the surfaces are at $77^{\circ}$ and $20^{\circ} \mathrm{K}$, respectively, adding powder may actually increase the heat transfer by providing paths of solid conduction. The radiant-heat transfer between the surfaces at these temperatures is so small that its elimination by adding powder is of little consequence. Radiation from a surface at $77^{\circ} \mathrm{K}$ is only about 0.004 of that from an identical surface at $300^{\circ}$ $\mathrm{K}$, because it is proportional to the fourth power of the absolute temperature. Of course the thickness of the insulating space must be considered in such a comparison. It is probably more correct to say that for boundaries at $77^{\circ}$ and $20^{\circ} \mathrm{K}$ a rather large thickness of powder would be required to compete with high-vacuum insulation.

It appears then that evacuated-powder insulation is very desirable if the warm boundary is at ordinary room temperature and the insulating space is thick enough (10 $\mathrm{cm}$ or more) so that solid conduction through the powder is not serious.

Of course, evacuated-powder insulation is not new. It has been used for many years in large industrial containers for liquid oxygen and liquid nitrogen. It is particularly suitable for large containers. Now that liquid hydrogen is being stored and transported in large quantities, evacuated powder appears attractive as the insulation to use in large liquidhydrogen vessels. Evacuated powder has been used in conjunction with a liquid-nitrogen-cooled shield for some vessels holding 750 liters of liquid hydrogen, and there are now under construction some liquid-hydrogen vessels of 6,000-liter capacity that utilize only powder insulation (no refrigerated shield).

\section{Dependence of the Rate of Evaporation Upon the Size and Shape of the Vessel}

It is quite easy to formulate for liquefied-gas containers some general relationships between the size and shape of the vessel and the rate of evaporation of the liquid it contains. First, because the heat leak into the container increases as the surface area increases, the most favorable shape is the sphere (the greatest ratio of volume to surface). The sphere also has some advantages of mechanical strength but in large sizes it may be expensive to fabricate. The next most favorable common shape is the square cylinder in which the length equals the diameter. The practical approach to this shape is the cylinder with standard commercial dished or elliptical heads. This design has good mechanical strength and is readily fabricated. It is stronger and has a somewhat larger ratio of volume to surface than the cylinder with the flat ends. However, in many cases, particularly vessels that are to be trans.ported by rail or highway, the maximum diameter is fixed.

Thus most of the designs for large insulated tanks for storing and transporting liquefied gases can be assigned to two categories: (1) fixed shape and (2) fixed diameter. It is of interest to see how the performance of such containers varies with the size of the vessel. The effectiveness of a container for liquefied gas is commonly given as the fraction (or percent) of the total contents that evaporates in 1 day.

\subsection{Vacuum-Insulated Vessels}

For vacuum-insulated containers of fixed shape, that is, containers of geometric similarity but of dif-ferent sizes, the heat leak by radiation and residualgas conduction is proportional to the surface area of the inner container, that is, proportional to $L^{2}$, where $L$ is a linear dimension of the container. The heat leak through the supports is also proportional to $L^{2}$, because the supports will have a length proportional to the linear dimension and a total cross-sectional area proportional to the volume (and weight) of the inner container. Then the total rate of heat leak is

$$
W=C L^{2}=C_{1} V^{(2 / 3)},
$$

where $C_{1}$ is a constant and $V$ is the volume of the inner container. The fractional rate of evaporation is

$$
\frac{W}{L_{v} \rho V}=\frac{C_{1} V^{(2 / 3)}}{L_{v} \rho V}=\frac{C_{1}}{L_{v} \rho V^{(1 / 3)}},
$$


where $L_{v}$ is the heat of vaporization of the liquid and $\rho$ its density.

\subsection{Vessels With Porous Insulation}

For containers insulated with porous materials, e. g., evacuated powders, the situation is somewhat different because, for similar geometry, the thickness, and therefore the effectiveness of the insulation, is proportional to the linear dimension of the container. The relation between the size and the performance of such vessels is not simple unless the heat conducted by the supports can be neglected. In this case the total heat leak is proportional to the linear dimension, so the fractional evaporation is proportional to $1 / \mathrm{V}^{(2 / 3)}$. Thus it is seen that for vessels utilizing this type of insulation, large size is even more advantageous than it is for high-vacuuminsulated vessels.

\subsection{Vessels of Fixed Diameter and Different Lengths}

The case of the container for rail or road transportation, in which the maximum diameter cannot exceed a certain value but the length may be varied, can be conveniently analyzed on the assumption that the ends are spherical, so its shortest length and minimum volume are those of a sphere having the permitted diameter. In this case the thickness of the insulation is greatly restricted, so it will be assumed that the thickness is constant. This means that the heat leak through the insulation will be proportional to the surface area both for high-vacuum insulation and for porous insulation. It. should be emphasized that this does not imply that the effectiveness of the various insulations is the same. It only means that in this circumstance the heat leak for unit area is constant for a given type of insulation and is not a function of the total area. Then, if we again ignore the heat leak through the supports,

$$
W=C\left[4 \pi r^{2}+2 \pi r(L-2 r)\right],
$$

where $C$ is a constant, $r$ is the maximum permissible radius, and $L$ the length of the container. The fractional evaporation is

$$
\frac{W}{L_{v} \rho V}=\frac{2 C L}{L_{v} \rho r\left(L-\frac{2}{3} r\right)} .
$$

As one would expect, the fractional evaporation changes very little with length, decreasing by only one-third when the length of the container is changed from $2 r$ to infinity.

\subsection{Comparison of Evaporation Rate Versus Size for Different Vessels}

Figure 2 shows graphically how the fractional rate of evaporation depends upon the volume of the container for the three conditions just discussed. It should be pointed out that the three curves should be compared with each other in regard to trend, not in regard to magnitude. The actual rates of

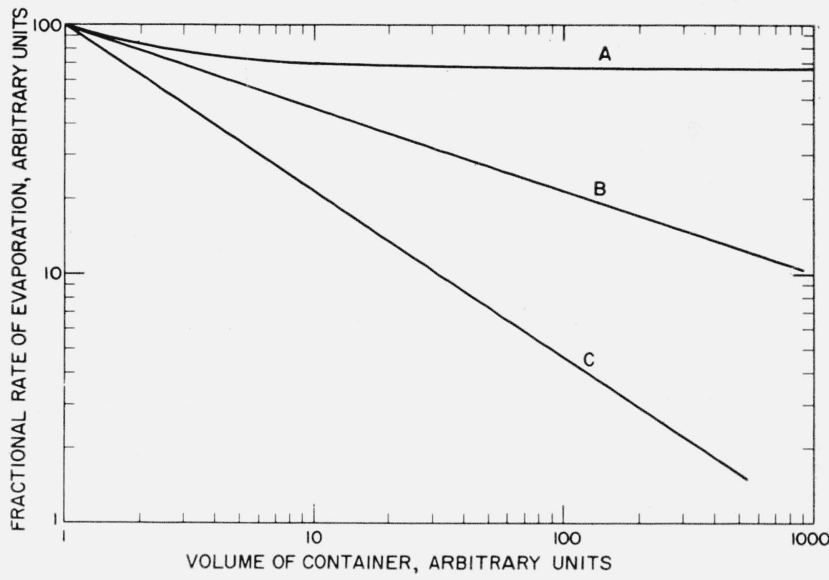

FIgURE 2. Dependence of the evaporation rate upon the size of the vessel.

A, Vessels of fixed diameter and various lengths; B, high-vacuum-insulated containers or containers having constant insulating thickness and similar geom etry; C, powder-insulated vessels of similar geometry throughout, including a constant ratio of insulating thickness to the other linear dimensions of the vessel.

evaporation can be determined only by computing the heat leaks for the particular insulation being used.

\subsection{Convenient Formula for Porous Insulation}

A good approximation for the heat transfer through a porous insulation of constant thickness in a vessel of conservative shape is given by

$$
W=\frac{\bar{k}\left(T_{2}-T_{1}\right)}{t} \times \sqrt{A_{1} A_{2}},
$$

where $\bar{k}$ is the mean effective thermal conductivity between the tempertaure $T_{2}$ and $T_{1}, T_{2}$ is the temperature of the outer surface, $T_{1}$ is the temperature of the inner surface, $A_{1}$ and $A_{2}$ are the areas of the inner and outer surfaces respectively, and $t$ is the thickness of the insulation. This formula is exact for concentric spheres. For cylinders with spherical, dished, or elliptical heads it has been estimated that the formula will give results within 5 percent if the thickness of the insulating space is not more than 50 percent of the radius of the cylinder. It is correspondingly reliable for other shapes in which the thickness of the insulation is a small fraction of the minor perimeter of the vessel. In reporting values of the thermal properties of insulators, the mean effective thermal conductivity is of ten given between commonly encountered temperatures. If the available data are presented in the more general form of instantaneous conductivities as a function of temperature, the mean effective thermal conductivity can be obtained from the relation

$$
\bar{k}=\left[\int_{T_{1}}^{T_{2}} k d T\right] /\left(T_{2}-T_{1}\right),
$$

where $k$ is the thermal conductivity at temperature $T$. If $k$ is a linear function of $T, \bar{k}$ is the arithmetic mean conductivity between $T_{1}$ and $T_{2}$. 


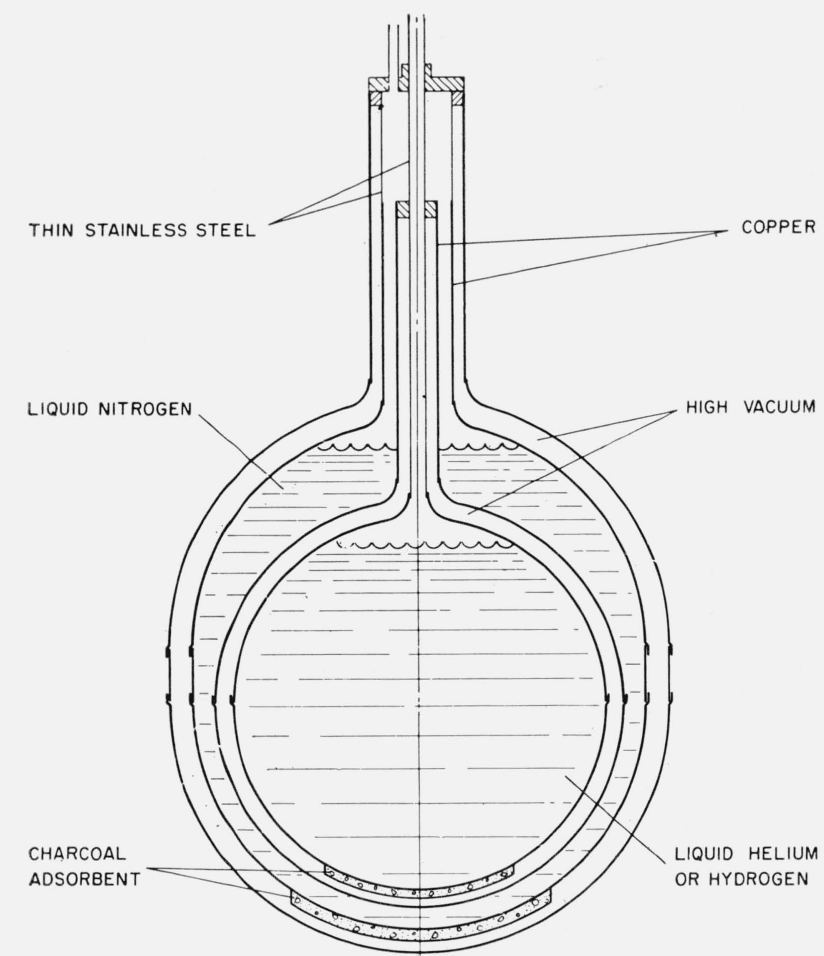

Figure 3. Simplified cross section of a Dewar vessel with a liquid-nitrogen-cooled shield.

\section{Shield Cooled With Liquid Nitrogen}

The vessels described in references [1] to [6] use liquid nitrogen to reduce the rate at which heat reaches the liquid helium or hydrogen being stored. In some cases the Dewar is simply immersed in liquid nitrogen contained in a larger vessel. Other designs are of unit construction, like that shown in figure 3 , in which there is a liquid-nitrogen-cooled shield suspended in the vacuum between the inner container and the warm wall. Most of the heat entering from the exterior is absorbed by the relatively inexpensive liquid nitrogen. This design is the most effective yet produced and is the most practical for storing small quantities of liquid hydrogen and helium. However, as the size of the vessel increases, the fractional evaporation rate without the shield decreases, and eventually a point is reached at which the extra initial cost of the liquid-nitrogen-cooled shield and the cost and bother of supplying liquid nitrogen are not worth the extra saving of the liquid being stored. This change-over point depends on the relative costs and heats of vaporization of liquid nitrogen and the liquid being stored.

\section{Vapor-Refrigerated Shields}

The vapor leaving the inner container of an insulated vessel is normally at a temperature very near the boiling temperature of the liquid being stored. This cold vapor constitutes a source of refrigeration that can be used to absorb some of the heat leaking into the vessel. In most vessels some of this refrigeration is utilized more or less accidentally in absorbing some of the heat which flows by solid thermal conduction down the vent pipe. An analysis of this process will be given later.

A way to make good use of the refrigeration available in the escaping vapor is to have it cool a shield suspended between the inner liquid container and the outer warm wall of the vessel. This device can be applied to high-vacuum-insulated vessels, as well as vessels utilizing evacuated-powder insulation. The openings in the shield required to admit piping and supports or reentrant irregularities that may be produced by attaching piping to one side of a shield will have little effect when powder insulation is used, but when the shield is in an empty space these openings and irregularities may somewhat reduce the effective over-all reflectivity of the shield. Moreover, in a vessel having sufficient room between the walls to accommodate a shield, the insulation thickness may be great enough to show a substantial improvement when powder is used. Because of the simpler construction and the fact that the ideal is more nearly approached, it is believed that the vaporcooled shield is more aptly applied to a vessel with evacuated-powder insulation.

Some commercial powder-insulated vessels make partial use of the cold escaping vapor by having it flow through several turns of pipe that lie between the inner and outer walls. It is believed, however, that the available refrigeration can be used more effectively if it is made to cool a shield located at the optimum position between the inner and outer walls of the insulating space.

The following analysis presents the general method of determining the optimum location of a vaporcooled shield and the expected gain in performance. The results are applied to the specific case of spherical containers for liquid hydrogen and helium with evacuated perlite insulation.

With evacuated-powder insulation filling the entire insulating space, the rate at which heat reaches the liquid is

$$
W_{1}=\frac{4 \pi k_{1} r_{1} r_{2}\left(T_{2}-T_{1}\right)}{r_{2}-r_{1}}=\dot{m} L_{v},
$$

where $k_{1}$ is the mean thermal conductivity of the powder in the temperature range $T_{1}$ to $T_{2}, r_{1}$ is the radius of the liquid container, $r_{2}$ is the radius of the vapor-cooled shield, $\dot{m}$ is the mass rate of evaporation, and $L_{v}$ is the heat of vaporization of unit mass of the liquid.

The rate at which heat reaches the shield is

$$
W_{2}=\frac{4 \pi k_{2} r_{2} r_{3}\left(T_{3}-T_{2}\right)}{r_{3}-r_{2}}=\dot{m} L_{v}+\dot{m} \Delta h,
$$

where $r_{3}$ is the radius of the outer boundary of the insulation, $k_{2}$ is the mean thermal conductivity of the powder in the temperature range $T_{2}$ to $T_{3}$, and $\Delta h$ is the change of specific enthalpy of the vapor as it is warmed from $T_{1}$ to $T_{2}$. 
Dividing (1) by (2) gives

$$
\frac{L_{v}}{L_{v}+\Delta h}=\frac{k_{1}}{k_{2}} \frac{\left(r_{3}-r_{2}\right)}{\left(r_{2}-r_{1}\right)} \frac{r_{1}}{r_{3}} \frac{\left(T_{2}-T_{1}\right)}{\left(T_{3}-T_{2}\right)} .
$$

Let $r_{3}=a r_{1}$ and $r_{2}=b r_{1}$, then

From (1),

$$
\frac{a(b-1)}{a-b}=\frac{k_{1}}{k_{2}} \frac{L_{v}+\Delta h}{L_{v}} \frac{\left(T_{2}-T_{1}\right)}{\left(T_{3}-T_{2}\right)}=g .
$$

$$
\frac{L_{v} \dot{m}}{4 \pi r_{1}}=k_{1} \frac{b}{b-1}\left(T_{2}-T_{1}\right)
$$

Because $b$ is a function of $a$ and $g$ only, and $g$ and $k$ are functions of $T_{2}$ only, $\dot{m} / r_{1}$ is a function of $a$ and $T_{2}$ only.

For each value of $a$ there is an optimum value of $\mathrm{T}_{2}$ and a corresponding optimum value of $b$, which will make $\dot{m} / r_{1}$ a minimum. Because, for a given insulation, $k_{1}, k_{2}$, and $g$ depend only upon the properties of the liquid and vapor, the fixed boundary temperatures $T_{1}$ and $T_{3}$, and the variable temperature $T_{2}$, we can compute values of $g$ for a series of temperatures $T_{2}$ in the range $T_{1}<T_{2}<T_{3}$ and use these g's for all subsequent computations dealing with the particular liquid being stored.

Then, for a given value of $a$, values of $\dot{m} / r_{1}$ can be computed and the minimum can be determined by graphical means. By plotting $\dot{m} / r_{1}$ versus the position of the shield, $(b-1) /(a-1)$, the location of the minimum will also yield the optimum value of $b$, the important design parameter. It will be noted also that for this vessel the rate of evaporation is proportional to the first power of the radius or to (volume) $)^{1 / 3}$, if heat conduction through supports is neglected. Thus this design offers the same premium for large size as that shown in curve $\mathrm{C}$ of figure 2 .

\subsection{Liquid-Hydrogen Containers}

A series of computations was made for values of $a$ ranging from 1.05 to 1.5 for a vessel containing liquid parahydrogen with evacuated-perlite insulation. The thermal conductivity of perlite is shown in figure 4, a curve based on two determinations

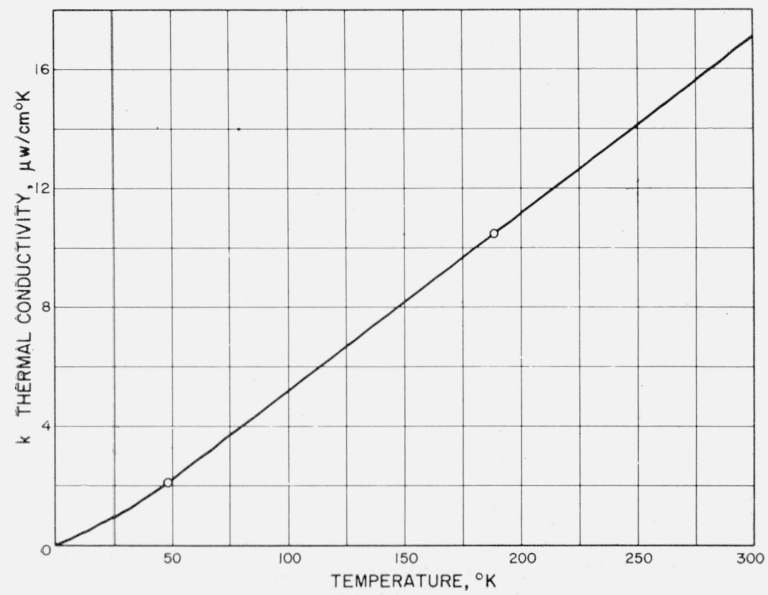

FIgURE 4. Thermal conductivity of -80 -mesh perlite. made at NBS, one giving the mean effective conductivity between $304^{\circ}$ and $76^{\circ} \mathrm{K}$, the other the mean conductivity between $76^{\circ}$ and $20^{\circ} \mathrm{K}$. These data are admittedly not as complete as they should be for the purpose at hand, but they will serve to demonstrate the usefulness of the vapor-cooled shield in this application. Errors arising from the linear interpolation used in figure 4 are not expected to exceed 20 percent. Additional measurements of the thermal conductivities of powders as functions of temperature are in progress at NBS. The values of $\Delta h$ were taken from the paper of Wooley, et al. [7].

Figure 5 shows the results of a representative computation for a liquid-hydrogen container. It is seen that at the optimum position of the shield, about 35 percent of the distance from the cold wall to the warm wall, the shielded vessel will have an evaporation rate of about 38 percent of the unshielded vessel. It is seen that the optimum location of the shield is not critical; the curve of $\dot{m} / \dot{m}^{*}$ has a very flat minimum. It was found that the effectiveness of the shield, $\dot{m} / \dot{m}^{*}$, at its optimum location is practically the same for values of $a$ ranging from 1.05 to 1.5 .

It will be noted that the shield is somewhat warmer than liquid nitrogen. The rate of evaporation will be lower if a liquid-nitrogen-cooled shield is used. The decision about the use of this method rather than liquid-nitrogen shielding will depend upon the relative costs of liquid nitrogen and liquid hydrogen and the cost of keeping the liquid nitrogen shield replenished. Also, the complete design study will include heat leaks from piping and supports, which cannot be resolved here because they are peculiar to the specific application and mechanical design.

It is remarkable also that the temperature of the shield and its optimum location, that is, its distance from the inner container divided by the distance between the inner and outer walls, $(b-1) /(a-1)$, are practically independent of the value of $a$. This is a consequence of the temperature dependence of the thermal conductivity of the powder and the specific heat of the vapor. As more complete data on the

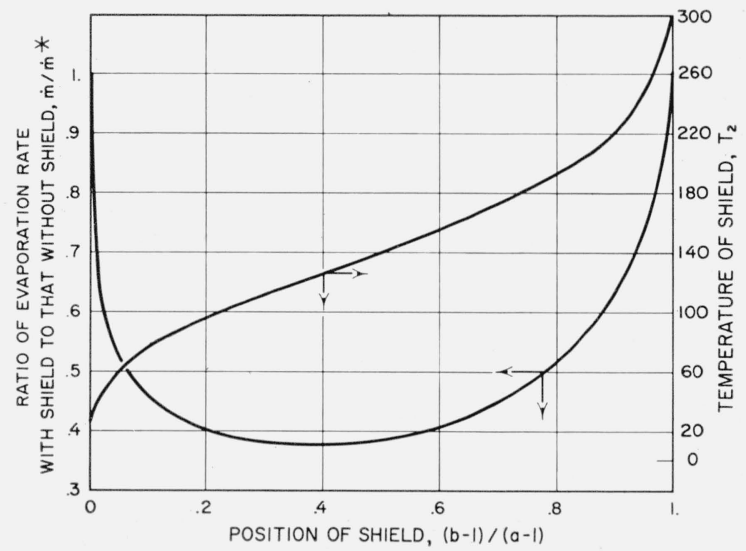

Figure 5. Optimum position and temperature of a vaporcooled shield in a powder-insulated vessel for liquid hydrogen. 
thermal conductivity of powder insulations become available, the optimum position should be recomputed.

To gain an idea of the performance that might be realized in a practical vessel, the results of these computations were applied to a spherical container for liquid hydrogen having an inner shell $2 \mathrm{~m}$ in outside diameter and an outer shell $3 \mathrm{~m}$ in inside diameter. This will have a capacity of slightly more than 4,000 liters. The evaporation rate resulting: from heat leak through evacuated-perlite insulation was found to be sufficient to evaporate 25 liters, or 0.62 percent, of the contents per day. A vaporcooled shield reduces this to 0.24 percent per day.

\subsection{Containers for Liquid Helium}

It is quite apparent that the effectiveness of the vapor-cooled shield depends upon the ratio of the heat of vaporization of the liquid being stored to the specific heat of the vapor. Accordingly the application of this device to the storage of liquid helium is very attractive because of the favorable ratio between the specific heat of the vapor and the heat of vaporization.

The results of computations for $a=1.5$ fully justified this optimism because it was learned that the vapor-cooled shield of a powder-insulated container for liquid helium at its optimum location will have a temperature of $62^{\circ} \mathrm{K}$, substantially lower than that of a liquid-nitrogen-cooled shield. The optimum position of such a shield was found to be $(b-1) /(a-1)=0.25$. The designer should again be cautioned against using this value after more data on the thermal conductivity become available. Measurements now in progress will undoubtedly yield more accurate data for this application.

Applying these results to the 4,000-liter spherical container described above, it was found that the heat leak through the insulation would cause liquid helium to evaporate at a rate of 7.7 percent per day from the unshielded vessel and 0.7 percent per day from the shielded vessel.

\subsection{Combination of High-Vacuum and Evacuated- Powder Insulation}

A logical refinement of the foregoing design is to use a high vacuum in the space between the coldest wall and the vapor-cooled shield, and powder insulation in the outer space. If the surfaces bounding the vacuum space have a low emissivity, this arrangement will result in a considerable improvement, gained at the expense of greater construction costs. The heat leak to the inner vessel, exclusive of solid conduction, can be computed by a method rather similar to that used earlier. In this case, however, the thickness of the high-vacuum space should be made as small as practical, so we can idealize the problem by assuming that the vacuum space has negligible thickness $\left(r_{1} \cong r_{2}\right)$. Then the rate at which heat is transmitted to the inner container by radia- tion from a shield at the temperature $T_{2}$ is

$$
\sigma \frac{e}{2-e} 4 \pi r_{1}^{2}\left(T_{2}^{4}-T_{1}^{4}\right)=\dot{m} L_{v},
$$

where $\sigma$ is the Stefan-Boltzman radiation constant, $e$ is the emissivity of the surfaces facing the vacuum space, $r_{1}$ is the radius of the inner container, $T_{2}$ and $T_{1}$ are the temperatures of the shield and inner container respectively, $\dot{m}$ is the mass rate of evaporation of the liquid being stored, and $L_{v}$ is its heat of vaporization. The heat that reaches the shield by conduction through the powder is

$$
\frac{k_{2} 4 \pi r_{1} r_{3}\left(T_{3}-T_{2}\right)}{\left(r_{3}-r_{1}\right)}=\dot{m} L_{v}+\dot{m} \Delta h,
$$

where $k_{2}$ is the mean thermal conductivity of the powder, $r_{3}$ is the radius of the outer shell at temperature $T_{3}$, and $\Delta h$ is the change in enthalpy of the vapor as it is warmed from $T_{1}$ to $T_{2}$. Dividing eq (4) by (5) and letting $a=r_{3} / r_{1}$ gives

$$
\frac{L_{v}}{L_{v}+\Delta h}=\frac{\sigma e r_{1}(a-1)\left(T_{2}^{4}-T_{1}^{4}\right)}{(2-e) k_{2} a\left(T_{3}-T_{2}\right)} .
$$

Because $\Delta h$ and $k_{2}$ are functions of $T_{2}$ that are not available from analytic expressions, the simplest method of solving this equation for $T_{2}$ is to use successive approximations. A general solution cannot be given. The equation must be solved for specific values of $r_{1}$ and $a$. For this reason the performance of the 4,000-liter vessel with an inner diameter of 2 $\mathrm{m}$ and an outer diameter of $3 \mathrm{~m}$, described earlier, was computed so that the results can be compared with those resulting from other insulation methods. It was found that with liquid hydrogen, the shield temperature will be $129^{\circ} \mathrm{K}$ and the evaporation rate will be 0.13 percent per day. When this system is used with liquid helium, the temperature of the shield is $86^{\circ} \mathrm{K}$ and the evaporation rate 0.33 percent per day. These computations assume an emissivity of 0.02 for the surfaces facing the vacuum space and ignore the heat leak through the supporting members.

\subsection{Discussion}

Table 1 shows the rates of heat transfer in terms of loss of liquid hydrogen and liquid helium in percent per day for several different types of insulation for a vessel of 4,000-liter capacity. These results do not take account of heat transfer by conduction through the supports nor heat conduction through the residual gas in a high vacuum. It should be emphasized that the designer and fabricator have a great amount of control over these latter sources of heat leak. The length of thermal path through insulating supports is particularly pliant to the ingenuity of the designer. Figure 6 gives a suggestion for increasing the length of supports.

The computations show that the effectiveness of the vapor-cooled shield in powder insulation, $\dot{m} / \dot{m}^{*}$, is practically independent of the thickness of the 
The outside diameter of the inner vessel is $2 \mathrm{~m}$ and, when powder insulation is used, the outer shell has an inside diameter of $3 \mathrm{~m}$. For the cases in which no powder is used, the diameter of the outer shell will be smaller. The emissivity of the surfaces facing the high-vacuum spaces is taken as 0.02 .

\begin{tabular}{|c|c|c|}
\hline \multirow[t]{2}{*}{ Insulation } & \multicolumn{2}{|c|}{$\begin{array}{l}\text { Evaporation rate caused by the heat flow through the insulation } \\
\text { (excluding conduction by solid supports and assuming no con- } \\
\text { duction by residual gas) }\end{array}$} \\
\hline & Liquid hydrogen & Liquid helium \\
\hline High vacuum a.... & $\begin{array}{l}\% / \text { Day } \\
4.0\end{array}$ & $\begin{array}{r}\% / \text { Day } \\
49.0\end{array}$ \\
\hline Evacuated perlite....... & 0.62 & 7.7 \\
\hline Evacuated perlite plus vapor-cooled shield........ & (shield temperature $=124^{\circ} \mathrm{K}$ ) & $\begin{array}{c}0.70 \\
\text { (shield temperature }=62^{\circ} \mathrm{K} \text { ) }\end{array}$ \\
\hline Evacuated perlite plus high vacuum a plus vapor-cooled shield _. & $\stackrel{.13}{\text { (shield temperature }=129^{\circ} \mathrm{K} \text { ) }}$ & (shield temperature $=86^{\circ} \mathrm{K}$ ) \\
\hline High vacuum a plus liquid $\mathrm{N}_{2}$ shield $\left(77^{\circ} \mathrm{K}\right) \ldots$ & .017 & .21 \\
\hline
\end{tabular}

a These are idealized conditions. The vacuum space is assumed to be negligibly thin and the vacuum is assumed to be so good that heat conducted by residual gas can be ignored.

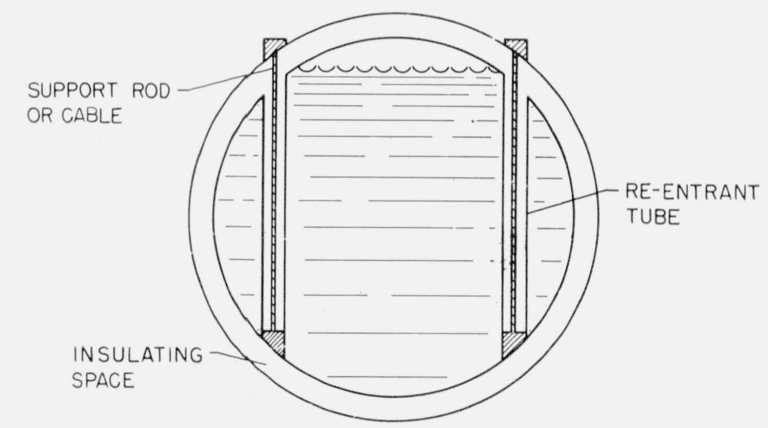

FiguRE 6. Reduction of thermal conduction through supports by increasing their length.

insulation, here measured by the constant $a$. The optimum location of the shield is slightly affected by the thickness of the powder. For the liquid-hydrogen vessel, the optimum position varied from 39 per cent of the way from the cold to the warm surface for $a=1.05$ to 32 percent of the way for $a=1.5$. The two computations for helium gave 30 percent for $a=1.2$ and 25 percent for $a=1.5$. These variations are rather trivial considering the flatness of the minimum in figure 5 .

The attainment of a first-rate high vacuum in an insulating space protecting the inner container requires first of all a completely leak-free construction, and also a thorough outgassing of all the metal surfaces bounding the high vacuum. The use of an adsorbent cooled to the temperature of the liquid being stored is highly recommended. The attainment of a superior vacuum is more easily accomplished in vessels for liquid helium storage than in liquid-hydrogen vessels because liquid helium provides a temperature at which all gases except helium have a negligible vapor pressure. Helium is not likely to be found in an insulating vacuum unless there is a leak. It has been observed that commercial containers with liquid-nitrogen-cooled shields have a much smaller heat leak when they are used to store liquid helium than when used with liquid hydrogen, probably because hydrogen gas is very thoroughly condensed when the container holds liquid helium. On the other hand, hydrogen is dissolved in, and is slowly released by, nearly all materials used to construct containers for cryogenic liquids, so provision should be made for its disposal if a high vacuum is to be maintained in vessels for other than helium.

A very obvious extension is the use of multiple shields. Because the cold vapor has a substantial refrigerative value after it leaves the shield discussed here, it is apparent that it could be utilized to cool additional shields.

Another source of refrigeration that could be utilized to cool the shield of a container for liquid parahydrogen is the heat absorbed by para-to-ortho conversion. If the proper catalyst is put into the vent tubes that cool the chield, the endothermic conversion to a higher ortho concentration will provide extra refrigeration. The cooling produced by converting hydrogen of 99.8 percent para (the equilibrium concentration at the normal boiling temperature) to 38.6 percent para (the equilibrium concentration at $100^{\circ} \mathrm{K}$ ) is 44 percent of the cooling produced by warming the gas from the hydrogen boiling point to $100^{\circ} \mathrm{K}$.

Finally, a word should be said about the storage of liquid oxygen and nitrogen. Because the ratio of the heat of vaporization to the change in enthalpy of the vapor upon being warmed to room temperature is much greater for these materials than it is for hydrogen and helium, it should be expected that the value of a vapor-cooled shield will be correspondingly less. However, the principles just discussed are equally valid for this application and should be given adequate consideration in the design of vessels for the long-term storage of liquid oxygen and nitrogen.

\section{Vapor-Cooled Vent Tube}

In those cases in which heat conduction through the supports or fill and vent tubes is a substantial part of the total, it may be worthwhile to use the escaping vapor to intercept part of the heat. This is an important consideration in the vent tubes of 
some Dewars. The escaping vapor maintains good thermal contact with the wall of the tube and absorbs a great deal of the heat that would otherwise enter and cause evaporation of the liquid being stored. The maximum possible saving from this process can be computed upon the assumption that the heat transfer between the vapor and the tube is perfect; that both have the same temperature at each level. Also, it is assumed that there is no lateral conduction or radiation to or from the tube. Because, in the temperature regions that are of interest, the thermal conductivity of the tube or support is a function of temperature, the practical solution of the problem can be simplified by assuming that this function is linear, that

$$
k=k_{0}+a\left(T-T_{0}\right),
$$

where $k$ is the thermal conductivity at the variable temperature $T, k_{0}$ is the thermal conductivity at the cold end, which has the temperature $T_{0}$, and $a$ is a constant. Then

$$
W=A\left[k_{0}+a\left(T-T_{0}\right)\right](d T / d x),
$$

which is the equation for heat conduction where $W_{0}$ is the heat current at any point in the tube, $A$ is the cross-sectional area of the tube, and $x$ is the distance from the cold end. Now the issuing vapor will absorb some of the heat traveling down the tube, so that

$$
W=W_{0}+\dot{m} C_{p}\left(T-T_{0}\right),
$$

where $W_{0}$ is the heat current that reaches the cold end of the tube, $\dot{m}$ is the mass rate of flow of the issuing vapor and $C p$ is its specific heat.

Upon combining eq (7) and (8) and integrating, it is found that

$$
\begin{aligned}
L / A=\left[1 /\left(\dot{m} C_{p}\right)\right] & \\
\times & {\left[a \Delta T+\left(k_{0}-W_{0} \frac{a}{\dot{m} C_{p}}\right) \ln \frac{W_{0}+\dot{m} C_{p} \Delta T}{W_{0}}\right], }
\end{aligned}
$$

where $L$ is the length of the tube and $\Delta T$ the temperature difference between the warm and cold ends.

The thermal conductivities of alloys commonly used for vent tubes of metal Dewars are rather complex functions of temperatures. However, eq (9) can be employed by dividing the total temperature interval into smaller intervals in which the thermal conductivity can be considered to be a linear function of temperature. Then eq (9) is applied to the lowest temperature interval, $\Delta T_{1}=T_{2}-T_{1}$, and the dependence of $W_{0}$ upon $L_{1} / A$ for various values of $\dot{m}$ is determined; $L_{1}$ is the length of tube lying in the lowest temperature interval. Then values of heat current $W_{0}^{\prime}$ at the bottom of the next higher temperature interval are determined by the relation

$$
W_{0}^{\prime}=W_{0}+\dot{m} C_{p} \Delta T_{1} \text {. }
$$

These values of $W_{0}^{\prime}$ are then used in eq (9) to com- pute values of $L_{2} / A$ for the second temperature interval, and the process is repeated as required. Finally, total values of $L / A$ are obtained by adding the individual values, $L_{n} / A$.

For a stainless-steel vent tube for hydrogen it is a sufficiently good approximation to use just two temperature intervals, from $20^{\circ}$ to $80^{\circ} \mathrm{K}$ and from $80^{\circ}$ to $300^{\circ} \mathrm{K}$. In figure 7 are plotted values of $W_{0}$

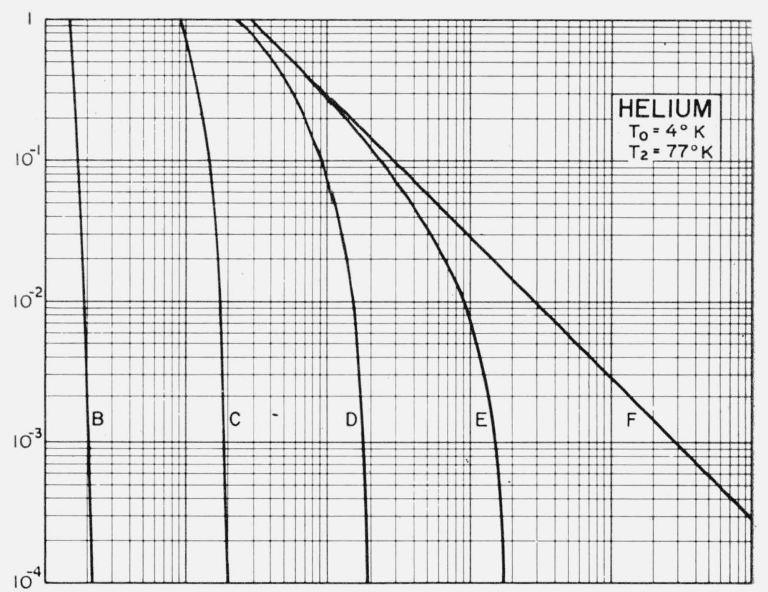

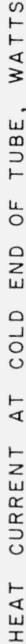

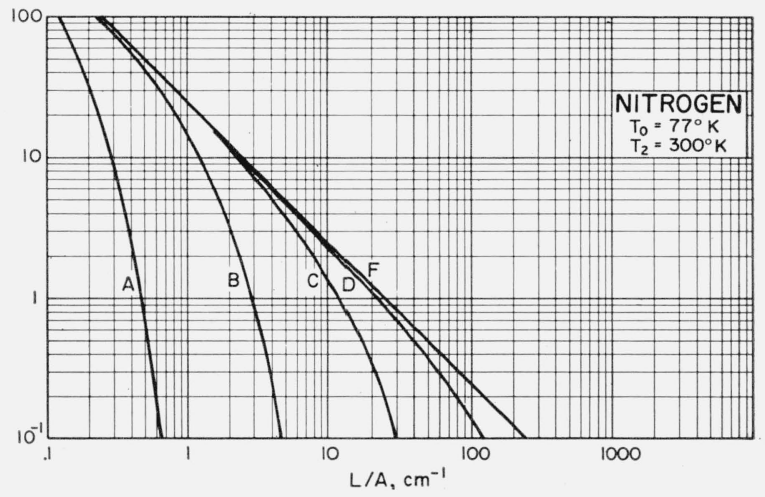

FIGURE 7. Results of computations of heat current reaching the cold end of a solid conductor counter to a stream of cold vapor.

A, $\dot{m}=1 \mathrm{~g} / \mathrm{sec} ; \mathrm{B}, 10^{-1} \mathrm{~g} / \mathrm{sec} ; \mathrm{C}, 10^{-2} \mathrm{~g} / \mathrm{sec} ; \mathrm{D}, 10^{-3} \mathrm{~g} / \mathrm{sec} ; \mathrm{E}, 1 \mathrm{0}^{-4} \mathrm{~g} / \mathrm{sec}, \mathrm{F}, \dot{m}=0$. 
as a function of $L / A$ for stainless-steel vent tubes. It should be remembered that these are limiting values, which assume complete heat exchange between the escaping vapor and the vent tube. In practical vessels, it may be necessary to provide baffles or other means for promoting heat transfer between the escaping vapor and the vent tube to approach the thermal equilibrium assumed for these computations. For hydrogen, which has a variable specific heat, $\dot{m} C_{p}$ can be replaced by $\dot{m}(\Delta h / \Delta T)$, where $\Delta h$ is the change of specific enthalpy for the temperature interval $\Delta T$. Values of thermal conductivities of stainless steel were taken from the data compiled by Powell and Blanpied [8].

\section{Conclusions}

The foregoing analyses show quite clearly that important benefits can be realized by utilizing the refrigerative value of the vapor which escapes from an insulated vessel for liquid hydrogen or helium. The problems were idealized in order to make their solution simpler; thus in many practical designs the values reported in this paper may be substantially altered by the mechanical requirements. However, there is some definite experimental evidence that these computed values are not unrealistic. A 50liter commercial container for liquid helium, similar to that illustrated in figure 3, protected with a liquid-nitrogen-cooled shield, showed an evaporation loss of 0.35 liter per day. If all this heat leak is attributed to thermal radiation from the shield at liquid-nitrogen temperature, the emissivities of the surfaces facing the vacuum space (assumed equal) are found to be 0.015 , which is an excellent value for copper, better than the 0.02 assumed in table 1 . This result implies that the heat conduction through residual gas and through the supporting neck of the Dewar was negligible. From the dimensions of the Dewar neck, $15.9 \mathrm{~mm}$ o.d., $0.5 \mathrm{~mm}$ thick, and $31.8 \mathrm{~cm}$ long $\left(L / A=130 \mathrm{~cm}^{-1}\right)$, figure 7 shows that if no vapor were escaping, the heat flow down the neck would be about $0.022 \mathrm{w}$, more than twice that required to cause the observed evaporation rate. However, with the observed rate of evaporation, 0.35 liter per day, $5.1 \times 10^{-4} \mathrm{~g} / \mathrm{sec}$, an interpolation ${ }^{3}$ of the curves of figure 7 shows that

3 The spacing of the lower parts of the curves of figure 7 indicates that logarithmic interpolation should be satisfactory in the region under consideration. the heat that actually reaches the cold end of the neck is less than $10^{-4} \mathrm{~W}$, a result consistent with the observations. Thus, it appears that a performance very near the ideal has been achieved in a relatively small vessel with a shield cooled with liquid nitrogen. Accordingly it should be entirely practical to approach the ideal in large vessels having either liquidnitrogen-cooled shields or vapor-cooled shields.

In many applications the neck of the 50-liter helium container described above is inconveniently small. It is possible to increase the neck diameter substantially, keeping the wall thickness and length the same, without seriously increasing the heat flow to the liquid helium. A value of $L / A$ of $80 \mathrm{~cm}^{-1}$ will give a heat current of very nearly $10^{-4} \mathrm{w}$, only 1 percent of the total heat leak. Doubling the diameter of the neck, making $L / A=65 \mathrm{~cm}^{-1}$, will resultin a heat current at the bottom of the neck of $2 \times 10^{-3} \mathrm{w}$, a 20 -percent increase. When the larger neck tubes are used, removable baffles should be provided, both to increase heat transfer between the escaping vapor and the tube, and to stop radiation down the tube.

The assistance of James A. Brennan in performing the tedious computations is gratefully acknowledged.

\section{References}

[1] W. F. Giauque, Rev. Sci. Instr. 18, 852 (1947).

[2] W. E. Henry and R. L. Dolecek, Rev. Sci. Instr. 21, 496 $(1950)$.

[3] A. Wexler, J. Appl. Phys. 22, 1463 (1951)

[4] H. L. Johnston, O. D. Gonzalez, and D. White, Rev. Sci. Instr. 22, 915 (1951).

[5] A. Wexler and H. S. Jacket, Rev. Sci. Instr. 22, 282 (1951).

[6] S. G. Sydoriak and H. S. Sommers, Jr., Rev. Sci. Instr. 22, 915 (1951).

[7] H. W. Wooley, R. B. Scott, and F. G. Brickwedde, J. Research NBS 41, 379 (1948) RP1932.

[8] Robert L. Powell and William A. Blanpied, NBS Circular 556 (1954).

Boulder, Colo., December 14, 1956. 